\title{
The thermodynamics of optical étendue
}

\author{
T Markvart \\ School of Engineering Sciences, University of Southampton, Southampton SO17 1BJ, UK
}

Received 2 June 2007, accepted for publication 12 November 2007

Published 12 December 2007

Online at stacks.iop.org/JOptA/10/015008

\begin{abstract}
The concept of étendue is applied to the propagation of luminescent radiation, and to the transformation of such radiation in absorbing and luminescent media. Central to this analysis is the notion of étendue as a measure of the number of rays in the beam which permits the definition of entropy and transition to the formalism of statistical mechanics. When considered from the statistical viewpoint, étendue conservation along the path of a beam in clear and transparent media then implies the conservation of entropy. The changes in thermodynamic parameters of a beam upon absorption and re-emission can then be determined in terms of the corresponding changes resulting from the addition or removal of photons from the incident and emitted beam. The thermodynamic theory which follows gives the rate of entropy generation in this process. At moderate light intensities, the results resemble the thermodynamics of a two-dimensional gas. The formalism allows an extension to absorption/emission processes where a high-temperature incident light beam is transformed reversibly into low-temperature luminescent radiation, corresponding to a potential increase in the open-circuit voltage of a solar cell.
\end{abstract}

Keywords: geometrical optics, thermodynamics

\section{Introduction}

Étendue is a convenient concept used in geometrical optics to describe the propagation of light through an optical system. For a beam with ray directions within a small solid angle $\delta \omega$ passing through an area $\delta A$, the element of étendue $\delta \mathcal{E}$ is defined as [1]

$$
\delta \mathcal{E}=n^{2} \cos \theta \delta \omega \delta A
$$

where $\theta$ is the angle between the direction of propagation and the normal to $\delta A$, and $n$ is the refractive index of the medium. A particularly useful aspect of étendue stems from the fact that the étendue of a beam propagating in a clear and transparent medium is conserved. In the analysis of light collecting systems, the conservation of étendue has played a key role in formulating the constraints to the limit of concentration of light and laying down the foundations for the theory of a non-imaging concentrator [1,2]. Étendue also acts as a measure of quantum states (or rays) in a beam [1] —an attribute that provides a natural link to statistical interpretation and thermodynamics. In particular, the conservation of étendue implies the conservation of entropy.

Radiation emitted by a black body has a zero chemical potential but radiation emitted in a restricted range on energies may have a non-zero chemical potential. For brevity, we shall call it quasi-black-body radiation. Luminescent radiation acquires the thermal characteristics of the emitter of this radiation through a detailed balance between absorption and emission, as expressed by the 'universal' relationship [3-5] between the absorption and fluorescent spectra. These relations can be viewed as an application of microscopic reversibility [6] to the energy exchange between the emitting substance and the luminescent radiation. When applied to a sequence of such elementary absorption/emission events, these relations guarantee a correct approach to thermodynamic equilibrium of both the substance and the radiation field.

Like scattering in classical mechanics, the transformation of one quasi-equilibrium beam into another introduces irreversibility, both from the point of view of optics [7] and thermodynamics, bringing about entropy generation. We shall show that the general result for the entropy generation resembles the familiar result of irreversible thermodynamics, and that the resulting entropy production rate complies inherently with the second law of thermodynamics by virtue of the conservation or increase of étendue in the absorption/emission process. For moderate radiation intensities, the formalism resembles the corresponding framework for a two-dimensional photon gas.

The plan of the paper is as follows. In section 2, we consider briefly the conservation of étendue from the statistical standpoint and show that this characteristic implies 
the conservation of all thermodynamic parameters of the beam. In section 3, we review the chemical potential of luminescent radiation from a thermodynamic point of view. Using the standard methods of irreversible thermodynamics, the results of section 3 are applied to the transformation of a high-temperature beam into low-temperature luminescence in section 4. Unlike previous treatments [8, 9], we shall describe this transformation not only in terms of the change of temperature of the beams but using also the change of chemical potential and the change of étendue.

The paper concludes with a brief discussion of a situation where the absorption/emission process is reversible, achievable, for example, in a device where electron-hole pairs, excited by the high-temperature incident beam, carry out work whilst being cooled to the ambient temperature, which is then used to increase the voltage generated by the solar cell.

\section{Entropy and the conservation of étendue}

Consider a beam of unpolarized light defined by the étendue (1), with frequency within a small interval $v \rightarrow v+\delta v$. A packet of photons in this beam which passes through the area $\delta A$ in a time interval $\delta t$ occupies a volume

$$
\delta V=\frac{c}{n} \cos \theta \delta A \delta t .
$$

Since the density of photon states per unit volume per unit solid angle per unit frequency is equal to $2 n^{3} v^{2} / c^{3}$ [10], the volume $\delta V$ contains $\left(\delta g_{v} \delta t\right)$ quantum states, where

$$
\delta g_{\nu}=\frac{2 n^{3} v^{2}}{c^{3}} \frac{\delta \nu \delta V \delta \omega}{\delta t}=\frac{2 v^{2}}{c^{2}} \delta \mathcal{E} \delta \nu .
$$

Since, in a clear medium, the étendue is conserved along the path of the beam, equation (3) implies that $\delta g_{v}$ is also conserved; this result can also be obtained by making use of the analogy between étendue conservation and the Liouville theorem of classical mechanics. The role of étendue as a measure of the number of rays in the beam has already been noted in [1], but equation (3) contains a new parameter not usually considered in geometrical optics: the frequency bandwidth $\delta v$, which becomes significant when considering processes which change the frequency of the beam.

In the absence of scattering, the number of photons $(\delta N \delta t)$ in the packet remains conserved, and so is therefore the photon distribution function $\rho_{v}$. It is then easily shown that equation (3) then implies the conservation of all extensive thermodynamic functions of the photon packet. Indeed, the energy transported by the beam

$$
\delta \dot{U}_{v}=h v \rho_{\nu} \delta g_{v}=\frac{B}{n^{2}} \delta \mathcal{E}
$$

is clearly also conserved. Equation (4) will be recognized as a statement of the brightness theorem: the ratio $B / n^{2}$, where $B$ is the brightness or radiance, is conserved along the path of the beam. In a similar manner, the entropy passing through $\delta A$ per unit time is equal to [8]

$$
\delta \dot{S}_{v}=\delta g_{v} k_{\mathrm{B}}\left\{\left(1+\rho_{\nu}\right) \ln \left(1+\rho_{\nu}\right)-\rho_{\nu} \ln \rho_{\nu}\right\},
$$

which is transparently also conserved. The conservation of other thermodynamic quantities, which can be defined through $\delta \dot{U}_{v}$ and $\delta \dot{S}_{v}$, now also follows.

Thus, the convective flow of any thermodynamic quantity remains constant in the absence of absorption or scattering. These flows cannot be equated to flows or currents in the meaning of irreversible thermodynamics (see, for example, chapter 3 , section 1 , of [6], which provides a particularly clear discussion of this point). However, the state parameters which are defined through these convective flows provide a basis for how irreversibility can be introduced-in a similar way as the usual formalism of irreversible thermodynamics is built from states of local thermodynamic equilibrium. That such basis states can be defined is possible by virtue of entropy, energy and photon number conservation for a beam in a clear medium. Since photons do not interact, these states does not correspond to a true thermal equilibrium but can, nevertheless, be characterized by well defined thermodynamic parameters such as temperature and chemical potential which remain constant as long as the beam propagates in a medium which complies with the assumptions of the étendue theorem. The use of convective flows as an analogue of equilibrium states to calculate the probability of fluorescence emission in a system with several apertures can be found in [11].

In section 4, we shall discuss how these thermodynamic quantities change as the beam transforms irreversibly during passage through an absorbing medium. First, however, we need to make a short detour to consider the chemical potential of light.

\section{Chemical potential of luminescent radiation}

It has been shown in section 2 that the thermodynamic functions remain constant for a beam of light propagating in a clear non-absorbing medium where the number of photons in the beam is conserved. We shall now consider how these functions change when photons are added to or removed from the beam.

By analogy with black-body radiation we can consider a cavity lined with a luminescent material at temperature $T_{0}$; for convenience we assume that the luminescence takes place, in a frequency range $v_{0} \rightarrow v_{0}^{\prime}$, with a quantum yield of unity. Any radiation, additional to the purely thermal radiation at temperature $T_{0}$, which has been introduced into the cavity within this spectral range will be repeatedly emitted and absorbed until it comes into thermal equilibrium with the luminescent material, and the occupation probability $\rho_{\nu}$ attains an equilibrium value

$$
\rho_{\nu}=\frac{1}{\mathrm{e}^{(h v-\mu) / k_{\mathrm{B}} T_{0}}-1}
$$

where the photon temperature is equal to the temperature of the medium $T_{0}$, and the photon chemical potential [12] is equal to the chemical potential $\mu$ of the electron-hole pairs. In the case of electro-luminescence the latter is often approximated in practice by the applied voltage [13].

In an alternative but equivalent arrangement, a volume $V$ with reflecting walls can contain an absorbing and luminescent 
medium. Luminescent photons will traverse the volume $V$ and be absorbed and re-emitted by the medium, until reaching thermal equilibrium. As discussed in section 1, the attainment of equilibrium is ensured by the detailed balance between absorption and emission [3, 4].

Let us now assume that the luminescent radiation can leave the cavity or volume $V$ through an aperture defined by an étendue $\mathcal{E}_{\text {out }}$. If the equilibrating absorption/emission processes are sufficiently fast, the radiation that emerges from the cavity has a spectrum described by (5). The number of photons $N$ in the volume is determined by the balance between the rate $\dot{N}_{\mathrm{p}}$ at which photons are supplied by the pump and the rate at which photons are emitted from the medium:

$$
\dot{N}_{\mathrm{p}}+\dot{N}\left(\mathcal{E}_{\text {out }}, T_{0}, 0\right)=\dot{N}\left(\mathcal{E}_{\text {out }}, T_{0}, \mu_{\text {out }}\right)
$$

where

$$
\dot{N}\left(\mathcal{E}_{\text {out }}, T, \mu\right)=\mathcal{E}_{\text {out }} \int_{v_{g}}^{v_{g}^{\prime}} \frac{2 v^{2}}{c^{2}} \rho_{\nu}(T, \mu) \mathrm{d} \nu=\frac{c \mathcal{E}_{\text {out }}}{4 \pi n^{3} V} N
$$

is the photon flux through the aperture and $N$ is the number of photons in volume $V$. The rate of photon generation in (6) includes the pump $\dot{N}_{\mathrm{p}}$ and thermal excitation from the surroundings, assumed to be at temperature $T_{0}$ of the medium.

In a similar fashion, the internal energy $U$ and entropy $S$ of photon gas inside volume $V$

$$
\begin{gathered}
U=V \int_{(v)} \frac{8 \pi v^{2}}{c^{3}} h \nu \rho_{\nu} \mathrm{d} \nu \\
S=V \int_{(\nu)} \frac{8 \pi v^{2}}{c^{3}} k_{\mathrm{B}}\left\{\left(1+\rho_{\nu}\right) \ln \left(1+\rho_{\nu}\right)-\rho_{\nu} \ln \rho_{\nu}\right\} \mathrm{d} v
\end{gathered}
$$

where $(v)=\left(v_{0}, v_{0}^{\prime}\right)$, are related to the convective energy and entropy flows $\dot{U}$ and $\dot{S}$ by

$$
\dot{U}=\frac{c \mathcal{E}_{\text {out }}}{4 \pi n^{3} V} U \quad \dot{S}=\frac{c \mathcal{E}_{\text {out }}}{4 \pi n^{3} V} S .
$$

Thus, the internal energy and entropy of the beam are related in a simple manner to the corresponding quantities of the photon gas in volume $V$. The same is, of course, true for other thermodynamic functions, in particular, for the free energy $F=U-T S$. The derivative of $F$ with respect to $N$ is the chemical potential:

$$
\mu=\left(\frac{\partial F}{\partial N}\right)_{V, T}=\left(\frac{\partial U}{\partial N}\right)_{V, T}-T\left(\frac{\partial S}{\partial N}\right)_{V, T}=u-T s
$$

where the energy $u$ and entropy $s$ per photon in volume $V$ are defined by

$$
u=\left(\frac{\partial U}{\partial N}\right)_{V, T} ; \quad s=\left(\frac{\partial S}{\partial N}\right)_{V, T} .
$$

Equations (10) and (11) refer to the quasi-equilibrium photon gas in volume $V$. By virtue of (9), however, they can be equally well written with reference to the emerging beam: all that needs to be done is to replace the thermodynamic functions

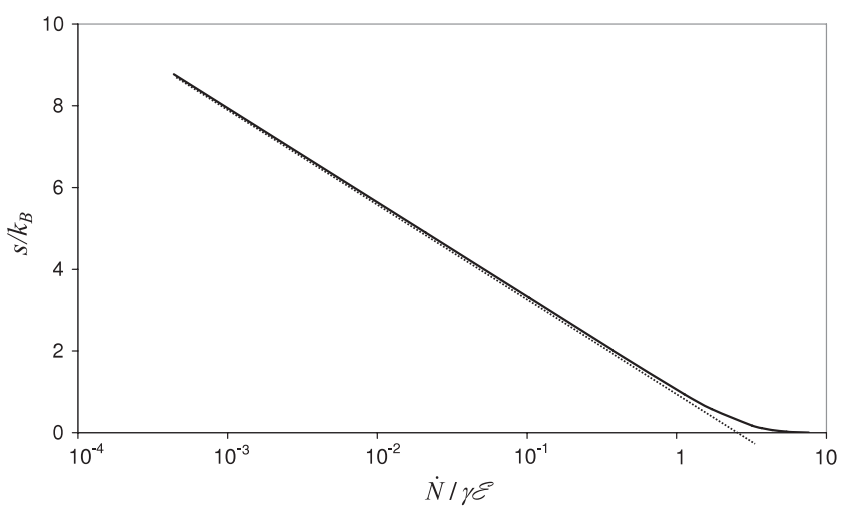

Figure 1. The entropy per photon $s$ (equation (11)), in units of the Boltzmann constant $k_{\mathrm{B}}$, as a function of the photon flux $\dot{N}$, in units of $\mathcal{E} \gamma\left(h v_{0}=1.42 \mathrm{eV}\right.$, corresponding to gallium arsenide). The dotted line gives the ideal-gas approximation (equation (15)).

$N, U, S$ by the corresponding flows, and taking the derivatives in (11) at constant étendue $\mathcal{E}$ :

$$
u=\left(\frac{\partial \dot{U}}{\partial \dot{N}}\right)_{\mathcal{E}, T} ; \quad s=\left(\frac{\partial \dot{S}}{\partial \dot{N}}\right)_{\mathcal{E}, T} .
$$

One can also define the analogue of pressure per photon by

$$
p=\left(\frac{\partial P}{\partial \dot{N}}\right)_{\mathcal{E}, T} .
$$

For a quasi-monochromatic beam of frequency $v_{0}$ and narrow bandwidth $\delta v$, the quantities (12) are analogous to the energy and entropy per photon considered in $[14,15]$ save for the appearance of the étendue $\mathcal{E}$ of the emitted beam

$$
u=h v_{0} ; \quad s=k_{\mathrm{B}} \ln \left(\frac{2 v_{0}^{2} \mathcal{E} \delta v}{c^{2} \dot{N}}+1\right) .
$$

The chemical potential is then easily obtained as

$$
\mu=h v_{0}-k_{\mathrm{B}} T \ln \left(\frac{2 v_{0}^{2} \mathcal{E} \delta v}{c^{2} \dot{N}}+1\right) .
$$

For radiation emitted within a broad frequency band, equations (12) provide a general recipe for the calculation of the energy and entropy change of a beam by the addition or a removal of a photon. They can be calculated without any difficulty numerically, and examples of these functions for an infinite frequency range $\left(v_{0}, \infty\right)$ (corresponding to a semiconductor of bandgap $h v_{0}$ ) are shown in figures $1-3$. The formalism becomes particularly simple for radiation of weak to moderate intensity when the probabilities $\rho_{v}$ are small. One can then readily show that

$$
\begin{aligned}
& \mu=h \nu_{0}+k_{\mathrm{B}} T \ln \left\{\frac{\dot{N}}{\mathcal{E} \gamma(T)}\right\} \\
& s(\mathcal{E}, T)=k_{\mathrm{B}} \ln \left\{\frac{\mathcal{E} \gamma(T)}{\dot{N}}\right\}+k_{\mathrm{B}} T \frac{\gamma^{\prime}(T)}{\gamma(T)} \\
& \cong k_{\mathrm{B}}\left\{\ln \left[\frac{\mathcal{E} \gamma(T)}{\dot{N}}\right]+1\right\}
\end{aligned}
$$

$u(\mathcal{E}, T)=h v_{0}+k_{\mathrm{B}} T^{2} \frac{\gamma^{\prime}(T)}{\gamma(T)} \cong h v_{0}+k_{\mathrm{B}} T$ 


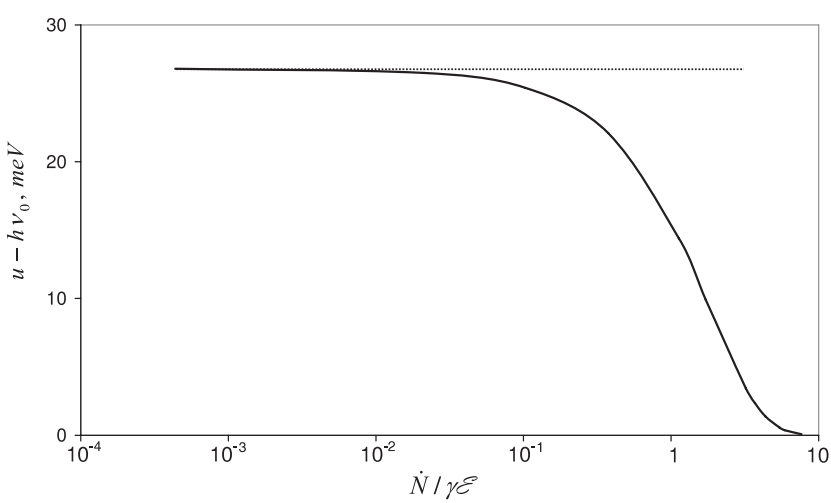

Figure 2. The difference $u-h v_{0}$ between the energy per photon (equation (11)) and the bandgap (full line) with the ideal-gas approximation (equation (16), dotted line). Other parameters as in figure 1.

where

$$
\gamma(T)=\int_{(v)} \frac{2 \nu^{2}}{c^{2}} \mathrm{e}^{h\left(\nu_{0}-v\right) / k_{\mathrm{B}} T} \mathrm{~d} \nu .
$$

For quasi-monochromatic radiation,

$$
\gamma=\frac{2 v_{0}^{2} \delta v}{c^{2}}
$$

which is independent of temperature. For radiation in a frequency band wider than $k_{\mathrm{B}} T / h$, on the other hand,

$$
\gamma(T)=\frac{2 v_{0}^{2} k_{\mathrm{B}} T}{h c^{2}}(1+\varepsilon)
$$

where $\varepsilon=2\left(k_{\mathrm{B}} T / h \nu_{0}\right)+2\left(k_{\mathrm{B}} T / h \nu_{0}\right)^{2}$ is a small correction if $h v_{0} \gg k_{\mathrm{B}} T$.

Equations (14)-(16) provide an interesting insight into the thermodynamics of a light beam: if the intensity of the beam is not too high, the entropy, energy and free energy of a photon added to the beam are given by the corresponding expressions for an ideal gas, where the étendue plays the role of volume. The product $\mathcal{E} \gamma(T)$ then plays the role of effective number of rays in the beam; for quasi-monochromatic beams considered in geometrical optics, $\gamma$ is constant, and the number of rays can be replaced simply by the étendue. If the correction $\varepsilon$ is neglected (results given by the approximate expressions in (15) and (16)), these thermodynamic functions coincide with the corresponding expressions for a two-dimensional ideal gas.

The formalism considered so far is independent of the excitation mechanism. Let us now suppose, for illustration, that the luminescence is excited by a beam of black-body radiation with temperature $T_{\text {in }}$ and étendue $\mathcal{E}_{\text {in }}$. Setting $\mu_{\text {in }}=0$ we can then write

$$
\begin{aligned}
\mu_{\text {out }} & =T_{0}\left\{\frac{\mu_{\text {out }}}{T_{0}}-\frac{\mu_{\text {in }}}{T_{\text {in }}}\right\}=T_{0}\left\{\frac{\mu\left(T_{0}, \mathcal{E}_{\text {in }}\right)}{T_{0}}-\frac{\mu\left(T_{\text {in }}, \mathcal{E}_{\text {in }}\right)}{T_{\text {in }}}\right\} \\
& -\left\{\mu\left(T_{0}, \mathcal{E}_{\text {in }}\right)-\mu\left(T_{0}, \mathcal{E}_{\text {out }}\right)\right\} .
\end{aligned}
$$

We make use of the relation

$$
\frac{u}{T^{2}}=-\frac{\partial}{\partial T}\left(\frac{\mu}{T}\right)_{\mathcal{E}},
$$

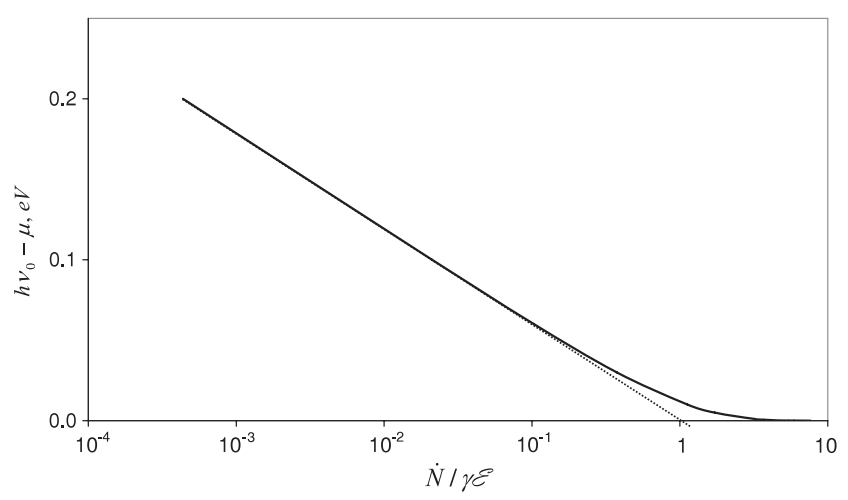

Figure 3. The energy separation between the bandgap $h \nu_{0}$ and the chemical potential $\mu$ (equation (10), full line), with the ideal-gas approximation (equation (14), dotted line). Other parameters as in figure 1.

which follows from standard thermodynamics but, in the spirit of (12), the derivative is taken at constant étendue rather than volume. The first term in the last equation (18) can then be transformed to $T_{0} \int_{T_{0}}^{T_{\text {in }}} \frac{u\left(\mathcal{E}_{\text {in }}, T\right)}{T^{2}} \mathrm{~d} T$.

Using the pressure $p$ per photon (13), the second term in (18) can similarly be transformed to $\int_{\mathcal{E}_{\text {in }}}^{\mathcal{E}_{\text {out }}} p \mathrm{~d} \mathcal{E}$. We now note that $\mathcal{E}_{\text {out }}$ cannot be smaller than $\mathcal{E}_{\text {in }}$ since, by time reversal symmetry, photons can always escape through the aperture whence they arrived. This integral is therefore always positive or zero if $\mathcal{E}_{\text {out }}=\mathcal{E}_{\text {in }}$.

Combining the two terms, we obtain

$$
\mu_{\text {out }}=T_{0} \int_{T_{0}}^{T \text { in }} \frac{u\left(\mathcal{E}_{\text {in }}, T\right)}{T^{2}} \mathrm{~d} T-\int_{\mathcal{E}_{\text {in }}}^{\mathcal{E}_{\text {out }}} p(\mathcal{E}, T) \mathrm{d} \mathcal{E} .
$$

Equation (19) determines the free energy or useful work that can be extracted by cooling a photon from temperature $T_{\text {in }}$ to $T_{0}$ if the étendue of the beam is expanded from $\mathcal{E}_{\text {in }}$ to $\mathcal{E}_{\text {out }}$ in the process. In practical applications, equation (19) gives the open circuit voltage of a solar cell solar, or the free energy difference that can be produced by light across the photosynthetic membrane.

The first term in (19) gives the maximum chemical potential achievable if the étendues of the incident and emitted beams are equal. This can be achieved by concentrating the incident beam with the use of lenses or mirrors, or by restricting the aperture of the emerging beam to the étendue of the incident beam. The second term in (19) represents a loss of free energy due to an increase in étendue during the beam transformation by the absorption/emission processes. It is interesting to note that this term resembles the loss of work upon expansion into vacuum, as can be seen by evaluating (19) in the ideal-gas approximation,

$\mu_{\text {out }}=\left(1-\frac{T_{0}}{T_{\text {in }}}\right) h v_{0}+k_{\mathrm{B}} T_{0} \ln \left\{\frac{\gamma\left(T_{\text {in }}\right)}{\gamma\left(T_{0}\right)}\right\}-k_{\mathrm{B}} T_{0} \ln \left(\frac{\mathcal{E}_{\text {out }}}{\mathcal{E}_{\text {in }}}\right)$

which can be interpreted as a particular case of the GouyStodola theorem [16]. The first term in (20) represents the Carnot efficiency of conversion of a monochromatic beam 
with frequency $v_{0}$, as discussed, for example, by Baruch et al for a two-level system [16]. The second term gives a correction on account of finite bandwidths of the absorption and luminescence spectra, equal to the entropy increase on cooling of an ideal gas from $T_{\text {in }}$ to $T_{0}$. The last term corresponds to a reduction in $\mu_{\text {out }}$ by expansion of the beam from $\mathcal{E}_{\text {in }}$ to $\mathcal{E}_{\text {out }}$, expressed in terms of the corresponding entropy change. A special case of (20) for specific solar cell geometries has been obtained in [18].

\section{Irreversible entropy production}

The emission of a photon beam by a volume of quasiequilibrium photon gas which was discussed in section 2 has been shown to be thermodynamically reversible since the entropy of a packet of photons in the convective flow emitted by the luminescent substance remains constant. As discussed in detail in [7], this reversibility manifests itself also in optical terms, as the original beam can be recovered by the use of optical instruments such as lenses or mirrors. In contrast, the transformation of a beam in an absorbing medium is, in general, irreversible, and can be understood in terms of the addition or removal of photons from the incident or emerging beams. This processes and the resulting entropy generation will now be discussed with the use of concepts that were introduced in section 3. We shall show that the resulting framework resembles closely the formalism of irreversible thermodynamics.

Suppose that a beam of quasi-black-body radiation at temperature $T_{\text {in }}$, étendue $\mathcal{E}_{\text {in }}$ and chemical potential $\mu_{\text {in }}$ in a frequency range $\left(v_{0}, v_{0}^{\prime}\right)$ is incident on a perfectly absorbing medium and is re-emitted with parameters $T_{0}, \mathcal{E}_{\text {out }}$ and $\mu_{\text {out }}$ in the same frequency range, where $T_{0}$ denotes again the temperature of the absorbing/luminescent medium. For complete generality, no assumption is made about the value of the chemical potential of the incident radiation, $\mu_{\mathrm{in}}$.

The entropy balance of the medium as a result of the absorption and emission processes has been discussed previously on a number of occasions (see for example [19] and [20]), and can be written, in the steady state,

$$
\left\{J_{S}(\text { in })-J_{S}(\text { out })\right\}+\frac{\dot{Q}}{T_{0}}+\frac{\mathrm{d}_{i} S}{\mathrm{~d} t}=0
$$

where $J_{S}\left(\right.$ in) and $J_{S}$ (out) are the incident and emerging entropy flows, $\mathrm{d}_{i} S / \mathrm{d} t$ is the rate of internal entropy generation, and $\dot{Q}$ is the net rate at which heat emitted by the luminescent medium is absorbed by a reservoir at temperature $T_{0}$. By virtue of energy conservation, this is equal to

$$
\dot{Q}=J_{U}(\text { out })-J_{U}(\text { in })
$$

where $J_{U}$ (in) and $J_{U}$ (out) are the energy flows in the incident and emerging beams. We have already indicated that the entropy and energy flows are associated with the creation and removal of photons from the beams, and are not equal to the convective entropy and energy flows $\dot{U}$ and $\dot{S}$ that were considered in section 2. Indeed, attempts to explain irreversible changes using the convective flows have led to apparent paradoxes which, to our mind, have not been satisfactorily resolved to date $[13,21]$.

Equation (21) can be transformed to a different form with the use of a standard relationship between the energy and entropy flows $J_{U}$ and $J_{S}$ of irreversible thermodynamics [6]:

$$
T J_{S}=J_{U}-\mu J_{N}
$$

where $J_{N}$ is the rate at which photons are supplied or removed from the system. Applying (23) to (21) with the use of (22) and the photon number conservation

$$
J_{N}(\text { in })=J_{N}(\text { out })
$$

we obtain

$$
\frac{\mathrm{d} S_{i}}{\mathrm{~d} T}=J_{U}\left(\text { in) }\left(\frac{1}{T_{0}}-\frac{1}{T_{\text {in }}}\right)-J_{N}\left\{\frac{\mu_{\text {out }}}{T_{0}}-\frac{\mu_{\text {in }}}{T_{\text {in }}}\right\}\right.
$$

where $J_{N}$ denotes the incident or emitted photon flux (24).

Equation (25) replaces the entropy and étendue conservation law for a beam propagating in non-absorbing media. Perhaps not surprisingly, it bears a close similarity to the entropy generation rate in irreversible thermodynamics. It is worth noting that (25) as been obtained far from equilibrium, in other words, for sizeable differences of the temperatures $T_{\text {in }}$ and $T_{0}$ and the chemical potentials $\mu_{\text {in }}$ and $\mu_{\text {out }}$. This implication of equation (25) is of interest in the broader context of losses in photovoltaic conversion, and is considered in a separate publication [22].

To be of practical value we need to define the flows $J_{\mathrm{U}} J_{S}$, and $J_{N}$. The photon flow is the rate $\mathrm{d} N / \mathrm{d} t$ of photon addition or removal from the photon gas or, equivalently, from the incident or emerging beams. Since all the incident photons are absorbed and all the emitted photons originate from luminescence in the medium, these flows can be defined by (6) for the rate of photon generation by the pump:

$$
\begin{gathered}
J_{N}(\text { out })=\dot{N}\left(\mathcal{E}_{\text {out }}, T_{0}, \mu_{\text {out }}\right)-\dot{N}\left(\mathcal{E}_{\text {out }}, T_{0}, 0\right) \\
J_{N}(\text { in })=\dot{N}\left(\mathcal{E}_{\text {in }}, T_{\text {in }}, \mu_{\text {in }}\right)-\dot{N}\left(\mathcal{E}_{\text {in }}, T_{0}, 0\right) .
\end{gathered}
$$

The energy and entropy flows resulting from photon creation or removal can then be written as

$$
J_{U}=\frac{\partial U}{\partial N} \frac{\mathrm{d} N}{\mathrm{~d} t}=u J_{N} ; \quad J_{S}=\frac{\partial S}{\partial N} \frac{\mathrm{d} N}{\mathrm{~d} t}=s J_{N}
$$

where $u$ and $s$ are the energy and entropy changes per photon which were introduced in section 3 . We note that, by virtue of photon conservation in the luminescent processes, the photon flows (26) are equal, and equation (23) follows from identity (10) in section 3 .

Dividing (25) by $J_{N}$ we obtain the entropy production $\sigma_{i}$ per photon:

$$
\sigma_{i}=u_{\text {in }}\left(\frac{1}{T_{0}}-\frac{1}{T_{\text {in }}}\right)-\left\{\frac{\mu_{\text {out }}}{T_{0}}-\frac{\mu_{\text {in }}}{T_{\text {in }}}\right\}
$$

where $u_{\text {in }}$ is the energy per photon in the incident beam. We shall now show that $\sigma_{i}$ is always positive or zero, as required by the second law of thermodynamics. To this end we can 
write, by a simple generalization of the argument leading to equation (21),

$$
\frac{\mu_{\text {out }}}{T_{0}}-\frac{\mu_{\text {in }}}{T_{\text {in }}}=\int_{T_{0}}^{T \text { in }} \frac{u\left(\mathcal{E}_{\text {in }}, T\right)}{T^{2}} \mathrm{~d} T-\frac{1}{T_{0}} \int_{\mathcal{E}_{\text {in }}}^{\mathcal{E}_{\text {out }}} p(\mathcal{E}, T) \mathrm{d} \mathcal{E} .
$$

Introducing $u_{\text {in }}=u\left(\mathcal{E}_{\text {in }}, T_{\text {in }}, \mu_{\text {in }}\right)$, expressing the difference $\left(1 / T_{0}-1 / T_{\text {in }}\right)$ as an integral of $1 / T^{2}$ then yields

$$
\sigma_{i}=\int_{T_{0}}^{T_{\text {in }}} \frac{u_{\text {in }}-u\left(\mathcal{E}_{\text {in }}, T\right)}{T^{2}} \mathrm{~d} T+\frac{1}{T_{0}} \int_{\mathcal{E}_{\text {in }}}^{\mathcal{E}_{\text {out }}} p(\mathcal{E}, T) \mathrm{d} \mathcal{E} .
$$

We have shown in section 3 that the second term cannot be negative. Since $u(T)$ is a non-decreasing function of $T$, the first term is positive or zero, giving $\sigma_{i} \geqslant 0$, as was to be demonstrated.

It is interesting to enquire about the maximum value of $\mu_{\text {out }}$ which is allowed on thermodynamics grounds. This maximum value $\mu_{\max }$, say, would be achieved in a reversible process with $\sigma_{i}=0$. Equation (27) then gives

$$
\mu_{\text {max }}=u_{\text {in }}\left(1-\frac{T_{0}}{T_{\text {in }}}\right) \cong\left(h v_{0}+k T_{\text {in }}\right)\left(1-\frac{T_{0}}{T_{\text {in }}}\right)
$$

where, for simplicity, we restrict attention to black-body incident radiation $\left(\mu_{\mathrm{in}}=0\right)$. Since luminescence is usually excited by radiation from a high-temperature source, the chemical potential (29) is greater than $h v_{0}-$ a situation that is clearly unacceptable from the viewpoint of Bose statistics (see equation (5)). This apparent quandary can be resolved if we imagine that the chemical potential $\mu_{\text {out }}$ is composed of two components, one associated with the emitted light and due to work being carried out by the medium. In other words, the hot-electron-hole pair generated by high-temperature photon beam carries out a certain amount of work (to be denoted by $w$ ) whilst being cooled to the temperature $T_{0}$ of the luminescent medium.

If such a term is included, the entropy generation becomes

$$
\sigma_{i}=u_{\text {in }}\left(\frac{1}{T_{0}}-\frac{1}{T_{\text {in }}}\right)-\frac{\mu_{\mathrm{out}}+w}{T_{0}} .
$$

The voltage $\left(\mu_{\mathrm{out}}+w\right) / q$ produced by such a 'hot-electron' solar cell is compared with the maximum voltage produced by a conventional cell (section 3 ) and the best currently achieved values in figure 4 . We should note that figure 4 shows the limit imposed by thermodynamics on a single-junction cell where energy absorbed by free carriers is, at least at open circuit, converted reversibly with maximum Carnot-cycle efficiency. This device-independent result can be compared with the hotcarrier converter proposed by Ross and Nozik [23] (see also reference [24]) which relies on the extraction of carriers from the hot-electron reservoir through selective contacts in narrow energy bands.

In the case of silicon with $h v_{0}$, for example, making use of the free carrier heat as suggested here can, in principle, increase the open circuit voltage by $76 \%$. If we assume that the rate of conversion in such a hot-electron cell follows the Shockley ideal solar cell characteristic, it can be shown without difficulty [22] that the use of reversible work provided by hot-electron cooling would raise the maximum achievable efficiency to $58 \%$.

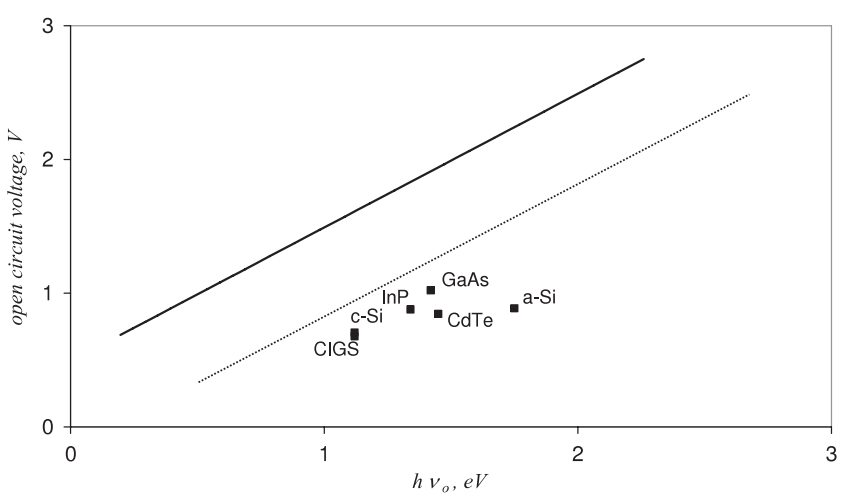

Figure 4. The maximum open circuit voltage of the hot-electron cell as a function of the bandgap $h v_{0}$, predicted by the present model (full line). The dashed line shows the voltage given by equation (20) for a black-body approximation to solar radiation $\left(T_{\text {in }}=6000 \mathrm{~K}\right.$;

$T_{0}=300 \mathrm{~K} ; \mathcal{E}_{\text {in }} / \mathcal{E}_{\text {out }}=2.18 \times 10^{-5}$ ). Points give the best measured values for different materials under one-sun illumination [23].

\section{Conclusion}

The concept of étendue, widely used in geometrical optics, has been applied to the propagation of luminescent radiation, and to the transformation of such radiation in an absorbing and luminescent medium. A beam of such radiation can be characterized by the temperature (equal to the temperature of the emitting substance), chemical potential, and the étendue. Central to this analysis is the notion of étendue as a measure of the number of rays in the beam which permits the definition of entropy and transition to the formalism of statistical mechanics.

We have shown that the transformation of a such beam of light upon absorption and re-emission can be discussed in terms of a thermodynamic theory which, at moderate intensities of light, resembles the thermodynamics of a twodimensional gas. We have obtained the rate of entropy generation of this process (equation (25)) and shown that it complies with the second law of thermodynamics.

The formalism allows an extension of the absorption/emission process to media where the elevated temperature of the photogenerated electron-hole pairs produces useful work which can be used to increase the voltage of solar cells. We have obtained the maximum value of this voltage which corresponds to a reversible transformation of a high-temperature light beam into low-temperature luminescent radiation.

\section{References}

[1] Welford W T and Winston R 1978 The Optics of Non-Imaging Concentrators (New York: Academic)

[2] Winston R 1970 J. Opt. Soc. Am. 60245

[3] Kennard E H 1918 Phys. Rev. 1129 Kennard E H 1926 Phys. Rev. 28672

[4] Stepanov B I 1957 Sov. Phys.-Dokl. 11281 Stepanov B I 1958 Izv. Akad. Nauk SSSR 221357

[5] van Roosbroeck W and Shockley W 1954 Phys. Rev. 941558

[6] deGroot S R and Mazur P 1984 Non-Equilibrium Thermodynamics (New York: Dover)

[7] Jones R C 1953 J. Opt. Soc. Am. 43138

[8] Landau L D 1946 J. Phys. (Moscow) 10503 
[9] Ries H 1981 J. Opt. Soc. Am. 72380

[10] See, for example Landau L D and Lifshitz E M 1958 Statistical Physics (Oxford: Pergamon)

[11] Markvart T 2006 J. Appl. Phys. 99026101

[12] For the definition of non-zero chemical potential of radiation see, for example Chel'tzov V F 1971 Solid State Commun. 91343

[13] Würfel P 1982 J. Phys. C: Solid State Phys. 153967

[14] Ross R T 1967 J. Chem. Phys. 464590

[15] Yablonovich E 1980 J. Opt. Soc. Am. 701362

[16] See, for example Bejan A 1988 Advanced Engineering Thermodynamics (New York: Wiley)
[17] Baruch P, Picard C and Swanson R 1981 Proc. 3rd European Photovoltaic Solar Energy Conf. (Dordrecht: Reidel) Baruch P 1985 J. Appl. Phys. 571347

[18] Ruppel W and Würfel P 1980 IEEE Trans. Electron Devices 27 877

[19] Weinstein M A 1960 J. Opt. Soc. Am. 50597

[20] Landsberg P T 1983 J. Appl. Phys. 542841

[21] deVos A and Pauwels H 1983 J. Phys. C: Solid State Phys. 166897

[22] Markvart T 2007 Appl. Phys. Lett. 91064102

[23] Ross R T and Nozik A J 1982 J. Appl. Phys. 533813

[24] Green M A et al 2002 Prog. Photovolt. 1055 